\title{
Granular Fingers on Jammed Systems: New Fluidlike Patterns Arising in Grain-Grain Invasion Experiments
}

\author{
S. F. Pinto, ${ }^{1}$ M. S. Couto, ${ }^{1}$ A. P. F. Atman, ${ }^{2}$ S. G. Alves, ${ }^{2}$ A. T. Bernardes,${ }^{3}$ H. F. V. de Resende, ${ }^{3}$ and E. C. Souza ${ }^{3}$ \\ ${ }^{1}$ Departamento de Física, Universidade Federal de Viçosa, 36570-000, Viçosa, MG, Brazil \\ ${ }^{2}$ Departamento de Física, Universidade Federal de Minas Gerais, Av. Antônio Carlos, 6627, CP 702, 31270-901, Belo Horizonte, MG, \\ Brazil \\ ${ }^{3}$ Departamento de Física, Universidade Federal de Ouro Preto, Campus Morro do Cruzeiro, 35400-000, Ouro Preto, MG, Brazil
} (Received 28 April 2006; published 6 August 2007)

\begin{abstract}
In this Letter we report spontaneous pattern formation in dense granular assemblies confined to a HeleShaw cell and quasistatic regime. Varied unexpected patterns, ranging from rounded to fingered, are observed due to the displacement of one granular material by another. Computer simulations reproduce the major features observed in these experiments. Two mechanisms are responsible for the pattern formation: crystallization of the injected grains and plastic deformation of the displaced grains. The experiment suggests analogies with viscous fingering and jamming transition experiments.
\end{abstract}

Granular materials are ubiquitous in nature as well as in daily life and in industry. Generally, they consist of discrete macroscopic grains large enough to make Brownian motion irrelevant. When subjected to large enough driving forces, they may exhibit a transition from solid to liquid and various patterns arise [1,2]. Pattern formation in granular systems has been the subject of several experimental and theoretical studies [2-4] that have been devoted to study its dynamical aspects using dilute granular systems and, up to now, has not been observed in dense granular systems. These works analyze different forms of energy injection (in systems far from equilibrium), focusing on dynamical processes leading to the spontaneous emergence of spatial structures [5-9].

Dilute granular systems - often described as "granular gases" or "granular liquids" because the material behavior is dominated by interparticle collisions due to the shaking or shearing - are successfully modeled using a kinetic theory framework [10]. However, a quantitative theory for dense granular systems is far less developed [11]. The behavior of such systems is dominated by prolonged contacts between particles, leading to formation of force-chain network along the material $[12,13]$, which causes significant changes in the bulk properties of the material, with important consequences for sound transmission [14], force and stress propagation [15,16], jamming [17-19] and memory effects [20]. A considerable effort has been made in the recent years to settle the question of stress propagation in dense granular systems.

The rheology of granular systems is a well-established field of research, as a long chain of papers published in the last decades demonstrate $[11,21,22]$. However, only recently the mechanisms underlying pattern formation in these systems have been experimentally and theoretically studied, particularly those concerned to the relation between particle mobility and the structure, in the quasistatic regime $[17,18]$.
In this Letter, we present an effort to understand a new phenomenon of pattern formation due to grain-grain displacement in dense granular assemblies through an experimental and numerical study of the granular fingering in a Hele-Shaw cell. The Hele-Shaw cell is used extensively to study pattern formation in fluid-fluid displacement via the well-known Saffman-Taylor instability [23-25]. In the case of granular materials, where the surface tension plays no role, there is no theoretical framework available to describe the mechanism of this peculiar type of pattern formation.

The experimental setup consists of grains confined to a cell formed by a pair of plane parallel glass plates of dimensions $(1.3 \times 1.3 \times 0.01) \mathrm{m}$ (lower plate) and $(0.8 \times$ $0.8 \times 0.01) \mathrm{m}$ (upper plate). The experiments were performed in a radial geometry, injecting the grains through a hole of $6 \mathrm{~mm}$ diameter at the center of the upper plate. The grains are inserted manually with the aid of a cylindrical teflon (TFE) rod whose diameter matches exactly with the hole in the upper glass plate. The particles are inserted one by one, and typically displace three beads on the substrate at the beginning of the injection. The injected grains never reach the cell boundaries. Initially, a circular monolayer of one type of grain is placed on the lower plate of the cell, which is then closed with the upper plate. The second type is injected grain by grain from above in a quasistatic manner. Experiments were performed using several combinations of grains and different grain sizes. We tested the effects of the preparation of the initial monolayer (ordered or random), and variation of the spacing between the plates (which was always larger than the larger grain used in each experiment). Varied patterns, ranging from almost circular to fingered ones were formed, depending on the size ratio of the grains and the cell spacing. Figures 1(a)-1(d) shows four stages of the development of a pattern formed by the injection of carbon steel spheres in styrofoam spheres. Very pronounced fingers were formed; tip splitting is evi- 
(a)

(b)

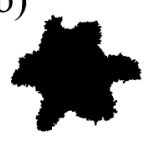

(c)

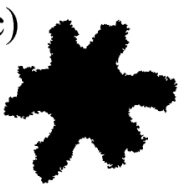

(g)

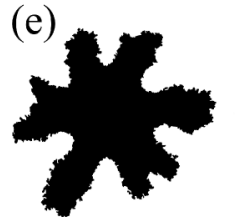

(f) (d)

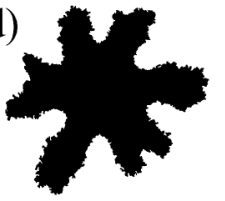

(h)
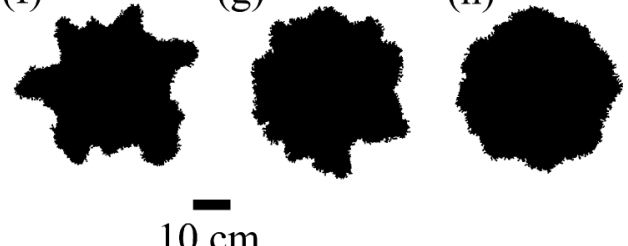

$10 \mathrm{~cm}$

FIG. 1. Patterns formed by the injection of carbon steel spheres $(3.94 \pm 0.03 \mathrm{~mm})$ in an initial monolayer of polydispersed styrofoam spheres (diameters ranging from 0.5 to $4.0 \mathrm{~mm}$ and average diameter of $2.9 \pm 0.6 \mathrm{~mm}$ ). (a) -(d) Development of the pattern for a cell spacing of $4.93 \mathrm{~mm}$. The number of grains injected corresponds, approximately, to 790 (a), 2760 (b), 4730 (c), and 6700 (d). (e)-(h) Transition from fingered to rounded pattern for different cell spacings: 4.93 (e), 5.17 (f), $5.44(\mathrm{~g})$, and $5.73 \mathrm{~mm}(\mathrm{~h})$. The patterns were formed after the injection of approximately 6700 carbon steel spheres.

dent in almost all of them. Patterns with fingers are formed only when the injected grains are bigger than those forming the initial monolayer, and the cell spacing is slightly larger than the diameter of the injected grain. If the cell spacing is increased the pattern changes from fingered to rounded, in an apparently continuous transition, as can be seen in Figs. 1(e)-1(h). It was observed that as the size of the injected grains is decreased in relation to the size of the grains in the initial layer, maintaining the cell spacing constant, the pattern becomes more rounded (Fig. 2).

Molecular dynamics (MD) [26] and probabilistic lattice gas automata (PLGA) [27,28] simulations have been performed trying to reproduce the experimental patterns. The results show that, depending on the parameters used to control the simulations, it is possible to obtain patterns

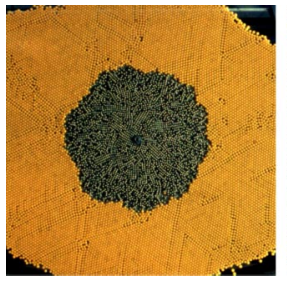

(a)

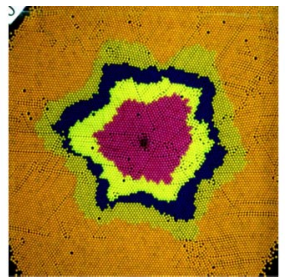

(b)

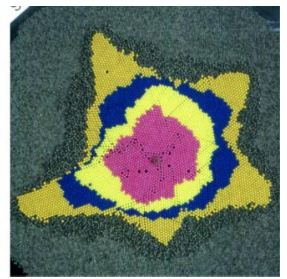

(c)
FIG. 2 (color online). Transition from rounded to fingered pattern as the ratio $R$ between the size of the injected grain and the size of the grain composing the initial layer is increased. (a) carbon steel injected in a polystyrene monolayer: $R=1 / 1.5$. (b) polystyrene injected in polystyrene: $R=1 / 1$. (c) polystyrene injected in carbon steel: $R=1.5 / 1$. The cell spacing is $6.3 \pm$ $0.1 \mathrm{~mm}$ and the largest grain has a diameter of $5.9 \pm 0.1 \mathrm{~mm}$. All figures are $60 \mathrm{~cm}$ wide. with all the morphologies obtained experimentally. In both cases, MD and PLGA, we performed simulations by using 2D disks, i.e., there is no superposition between disks, differently from that observed in some real 3D experiments.

In MD simulations, the force model used is essentially that of Cundall and Strack [29]: overlapping disks are coupled by both normal and tangential springs, of respective stiffness, $k_{n}$ and $k_{t}$. A linear viscous damping force (dashpot) acts in the normal direction (parallel to the line connecting the centers of the disks), with the damping coefficient chosen to correspond to critical damping. The tangential forces are limited by the Coulomb condition, with a coefficient of (both static and dynamic) friction, $\mu=0.5$. Besides the usual Kelvin-Voight rheology for the contacts, we introduced an additional parameter, $\eta$, ranging between 0 and 1 , in order to reproduce the energy loss $(0=$ no loss, $1=100 \%$ loss $)$ simulating the interaction of the grains with the cell plates. Since we have simulated the experiments using 2D disks, we opted to introduce this parameter as a "dragging" friction, correcting the velocity values in each MD step. Differently from that observed in experiments, we also obtain fingers when the injected disks are smaller than those in the initial monolayer, but only if the stiffness of injected particles is greater than the stiffness of the initial monolayer.

The PLGA is defined on a two-dimensional triangular lattice and disks of two sizes are used: the smaller ones with half the diameter of the bigger ones. Rules of movements were defined [30], mimicking mechanical properties of the material, mainly the resistance to the movement of the particles, due to the friction between particles and the plates, but also due to the jamming of particles. The injection of small disks in a monolayer of big disks results in a circular pattern. Fingers are formed only when big disks are injected in a monolayer of small ones. They move along the directions of the lattice. The resistance to their movement is given by the definitions of probabilities, discussed above, but also by geometrical restrictions, what does not occur to the previous case (smaller disks injected in a substrate of big disks). The geometrical aspects are very relevant, because the limited space for the big disks represent also the tension observed experimentally. Figure 3 shows some of the results obtained. So, simulations capture the basic features responsible for the pattern formation: crystallization of the injected grains and plastic deformation of the displaced grains.

Figure 4(a) shows a pattern formed by the injection of polystyrene spheres in carbon steel spheres. The Young's modulus of the two materials are $\approx 5$ and $\approx 200 \mathrm{GPa}$, respectively. The static friction coefficient between these materials and the glass plate of the cell are 0.69 and 0.19 , respectively. We observe that the injected spheres crystallize spontaneously in a triangular lattice presenting several grain boundaries. Examining the pattern, one can see that 


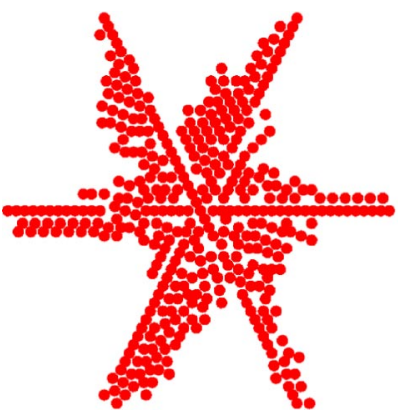

(a)

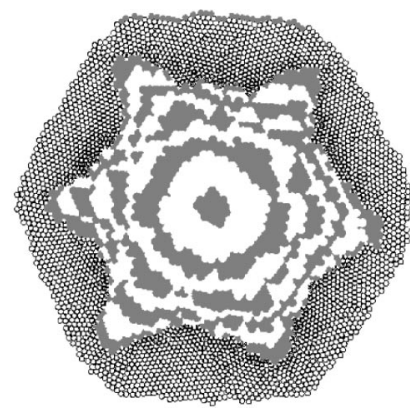

(b)
FIG. 3 (color online). Simulated patterns. (a) PLGA pattern formed by the introduction of 1700 big disks in a substrate of small disks. In this case a small probability of movement of the big disks was defined, representing a great resistance to their penetration (to allow better visualization, the small disks are not represented in the figure). (b) Typical MD pattern is showed. The disks at the initial layer are hollow and the injected disks are filled with white or gray - at each 250 particles the color is changed. The fingered pattern was observed for high values of the friction coefficient with the plates and for stiffness of injected disks 10 times greater than that used for the disks in the initial monolayer. In this figure we use $\mu=0.5$ for the friction coefficient between the disks, $k_{t} / k_{n}=0.75$, and $\eta=0.99$.

all fingers are oriented along a principal lattice direction, as shown in Fig. 4(b), while the regions between two adjacent fingers are oriented along a direction rotated $30^{\circ}$ from the fingers direction. This is observed, with very few exceptions, in all patterns exhibiting fingers. Along the fingers direction, the spheres are arranged such that a force applied to one sphere is transmitted directly to the next one, and so
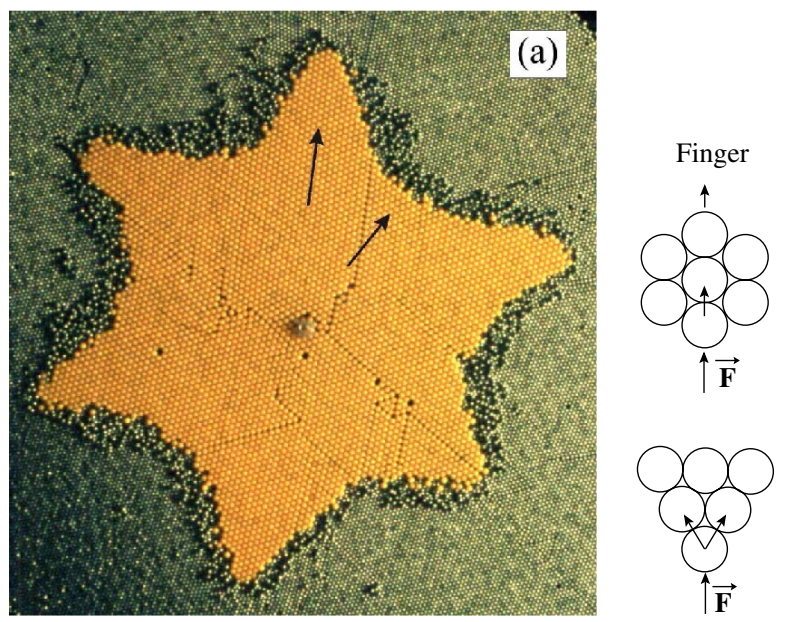

FIG. 4 (color online). Orientation of the spheres forming the pattern. The fingers seen in (a) are oriented along the direction shown in (b) while the region between two adjacent fingers is oriented along the direction shown in (c). The pattern was formed by injecting polystyrene spheres $(5.9 \pm 0.1 \mathrm{~mm})$ in carbon steel spheres $(3.94 \pm 0.03 \mathrm{~mm})$ with a cell spacing of $6.3 \pm 0.1 \mathrm{~mm}$ on all the way to the tip of the finger [Fig. 4(b)]. Thus, the movement of the spheres along this direction is preferred and the finger grows. In the region between two fingers the spheres are arranged in a manner that a force applied in one sphere is redirected along two different directions [Fig. 4(c)]. This inhibits the movement of the spheres along the original force direction and there is no finger.

In order to evaluate the real influence of crystallization in finger formation, we performed experiments in which the injected grains were a mixture of carbon steel spheres of two sizes. The ratio between the diameters of the larger and smaller spheres was $1.24 \pm 0.01$. The existence of spheres with two different sizes should avoid, or at least hinder, the formation of regions with an ordered triangular lattice. If the orientation of the spheres in these regions is the cause of finger formation, they should become smaller or completely disappear. Maintaining the cell spacing constant, the percentage of spheres of each size was varied. Figure 5 shows that the patterns present more pronounced fingers for injection of monodisperse spheres, while for a bidisperse mixture they become rounded.

One can also see in Fig. 4 that around the pattern there is a disturbed region formed by the grains in the initial monolayer. This happens in all experiments in which the injected grains are the larger ones since the cell spacing is large enough for the grains of the initial monolayer to move upwards, forming a thicker layer around the pattern. The border between this region and the initial monolayer has roughly the same shape as the pattern formed by the injected grains. Since these grains are in contact with both plates of the cell, the resistance to their movement is larger than in the rest of the monolayer and in the layer of injected grains. The thicker region therefore inhibits the movement of the injected grains. By analogy with the pattern phenomenon in fluid-fluid displacement, this region with higher resistance for front propagation would correspond to a fluid with high "viscosity", leading to the formation of a fingered pattern.

When the injected grains are smaller than the ones of the initial monolayer, the situation described above is reversed and the injected grains are the ones that move upwards,

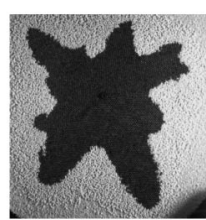

(a)

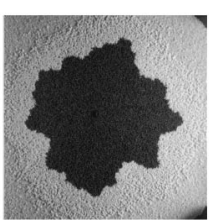

(b)

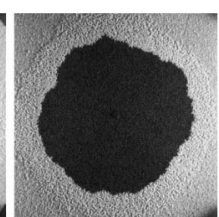

(c)

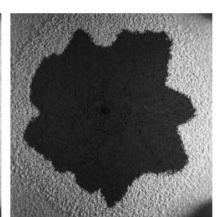

(d)
FIG. 5. Patterns formed by injecting a bidisperse mixture of carbon steel spheres in styrofoam spheres. The percentage of the larger carbon steel spheres in each experiment is: (a) $100 \%$, (b) $50 \%$, (c) $25 \%$, (d) $0 \%$. The mixture of spheres with two sizes hinders finger formation. In our experiments, size segregation between carbon steel spheres was not observed. All figures are $\approx 50 \mathrm{~cm}$ wide. 
inhibiting their crystallization. In this case, the viscosity of the injected grains is larger than that of the monolayer, and the finger formation is inhibited. When the spacing between the plates of the cell is increased all the grains can move upwards, leading to the formation of rounded patterns. It is worth emphasizing that this analogy is just an interpretation of the experimental findings, since there is no equivalent to viscosity in granular materials.

Tip splitting was observed only when using styrofoam as the displaced material. This is, probably, due to the permanent deformation of the styrofoam spheres, which increases even further the resistance to displacement, making the viscosity of the monolayer even larger than the one for rigid grains. So, although crystallization of the injected grains is essential for finger formation, the behavior of the initial monolayer plays an important role defining the finer details in the pattern formed.

Finally, our findings suggest a connection with the work of Goldenberg and Goldhirsch [31]. Our observations are in agreement with the models of force transmission in granular materials which consider stress propagation along the principal directions of the triangular lattice (hyperbolic model). So, fingered patterns might arise due to the stress propagation along these preferential directions, in which the grains are lined up. Rounded shapes, compatibles with elliptic models for force transmission were obtained for large spacing, which may be related to a lower stress field. These conjectures could be confirmed by using photoelastic grains in the experiments, and were already confirmed by the MD simulations - which we intend to present shortly together with a detailed theoretic description of the models.

We thank R. Dickman, P. Claudin, and H. J. Herrmann for discussions and suggestions. This work was partially supported by CNPq, CAPES, and FAPEMIG. We also are indebted to the referees by their enlightening suggestions.

[1] H. M. Jaeger, S. R. Nagel, and R. P. Behringer, Rev. Mod. Phys. 68, 1259 (1996).

[2] Physics of Dry Granular Media, edited by H. J. Herrmann, J.-P. Hovi, and S. Luding (Kluwer Academic, Dordrecht, 1998).

[3] I. S. Aranson and L. S. Tsimring, Rev. Mod. Phys. 78, 641 (2006).

[4] J. Duran, Sands, Powders, and Grains: An Introduction to the Physics of Granular Materials (Springer, New York, 2000).

[5] P. B. Umbanhowar, F. Melo, and H. L. Swinney, Nature (London) 382, 793 (1996).
[6] T.H. Metcalf, J.B. Knight, and H.M. Jaeger, Physica (Amsterdam) 236A, 202 (1997)

[7] O. Pouliquen, J. Delour, and S. B. Savage, Nature (London) 386, 816 (1997).

[8] F. Malloggi, J. Lanuza, B. Andreotti, and E. Clément, in Powders and Grains 2005, edited by R. Garc-Rojo, H. J. Herrmann, and S. McNamara (Taylor \& Francis, London, 2005).

[9] H. J. Herrmann and G. Sauermann, Physica (Amsterdam) 283A, 24 (2000).

[10] N. V. Brilliantov and T. Pöschel, Kinetic Theory of Granular Gases (Oxford University, Oxford, 2004).

[11] G. D. R. Midi, Eur. Phys. J. E 14, 367 (2004).

[12] J.P. Wittmer, P. Claudin, M. E. Cates, and J.-P. Bouchaud, Nature (London) 382, 336 (1996).

[13] J. Geng, G. Reydellet, E. Clément, and R. P. Behringer, Physica (Amsterdam) 182D, 274 (2003).

[14] C.H. Liu and S.R. Nagel, Phys. Rev. Lett. 68, 2301 (1992).

[15] J.-P. Bouchaud, P. Claudin, M. E. Cates, and J.P. Wittmer, in Physics of Dry Granular Media, edited by H.J. Herrmann, J.-P. Hovi, and S. Luding (Kluwer Academic, Dordrecht, 1998).

[16] P. Claudin and J.-P. Bouchaud, Phys. Rev. Lett. 78, 231 (1997).

[17] E. Kolb, J. Cviklinski, J. Lanuza, P. Claudin, and E. Clément, Phys. Rev. E 69, 031306 (2004).

[18] E. Kolb, C. Goldenberg, S. Inagaki, and E. Clément, J. Stat. Mech. (2006) P07017.

[19] A.L. Liu and S. Nagel, Nature (London) 396, 21 (1998).

[20] J. Geng et al., Phys. Rev. Lett. 87, 035506 (2001).

[21] R. A. Bagnold, Proc. R. Soc. A 225, 49 (1954).

[22] P. G. de Gennes, Physica (Amsterdam) 261A, 267 (1998).

[23] P. Meakin, Fractals, Scaling and Growth Far from Equilibrium (Cambridge University Press, Cambridge, England, 1998).

[24] D. A. Kessler, J. Koplik, and H. Levine, Adv. Phys. 37, 255 (1988).

[25] E. Ben-Jacob, R. Godbey, N. D. Goldenfeld, J. Koplik, H. Levine, T. Mueller, and L. M. Sander, Phys. Rev. Lett. 55, 1315 (1985).

[26] M.P. Allen and D. J. Tildesley, Computer Simulations of Liquids (Clarendon, Oxford, 1989).

[27] X. Sun, S. F. You, D. M. Wang, and Y. T. Yue, Int. J. Mod. Phys. C 16, 745 (2005).

[28] G. W. Peng and H. J. Herrmann, Phys. Rev. E 49, R1796 (1994).

[29] P. A. Cundall and O.D. L. Strack, Geotechnique 29, 47 (1979).

[30] T. Toffoli, Physica (Amsterdam) 10D, 117 (1984).

[31] C. Goldenberg and I. Goldhirsch, Nature (London) 435, 188 (2005). 